\title{
Low frequency noise from wind turbines mechanisms of generation and its modelling
}

\section{Aagaard Madsen, Helge}

Published in:

Journal of Low Frequency Noise, Vibration and Active Control

Link to article, DOI:

10.1260/0263-0923.29.4.239

Publication date:

2010

Link back to DTU Orbit

\section{Citation (APA):}

Aagaard Madsen, H. (2010). Low frequency noise from wind turbines mechanisms of generation and its modelling. Journal of Low Frequency Noise, Vibration and Active Control, 29(4), $239-251$.

https://doi.org/10.1260/0263-0923.29.4.239

\section{General rights}

Copyright and moral rights for the publications made accessible in the public portal are retained by the authors and/or other copyright owners and it is a condition of accessing publications that users recognise and abide by the legal requirements associated with these rights.

- Users may download and print one copy of any publication from the public portal for the purpose of private study or research.

- You may not further distribute the material or use it for any profit-making activity or commercial gain

- You may freely distribute the URL identifying the publication in the public portal

If you believe that this document breaches copyright please contact us providing details, and we will remove access to the work immediately and investigate your claim 


\title{
Low Frequency Noise from Wind Turbines Mechanisms of Generation and its Modelling
}

\author{
Helge Aagaard Madsen \\ Research Specialist, PhD. \\ Wind Energy Division
}

Risø DTU National Laboratory for Sustainable Energy, DTU

DK-4000 Roskilde, Denmark

E-mail: hama@risoe.dk

\section{Summary}

The objective of the present paper is to present an overview of LFN characteristics of modern MW turbines based on numerical simulations. Typical sizes of modern turbines are from 1-3 MW nominal generator power and a rotor diameter ranging from $80-100 \mathrm{~m}$ but larger prototypes up to $5 \mathrm{MW}$ and with a rotor diameter of $126 \mathrm{~m}$ have now been installed. The numerical investigations comprise the common upwind rotor concept but also the turbines with a downwind rotor are considered. The reason to include the downwind rotor concept is that this turbine design has some advantages which could lead to future competitive designs compared with the upwind three-bladed rotor.

The simulation package comprises an aeroelastic time simulation code HAWC2 and an acoustic low frequency noise (LFN) prediction model. Computed time traces of rotor thrust and rotor torque from the aeroelastic model are input to the acoustic model which computes the sound pressure level (SPL) at a specified distance from the turbine.

The influences on LFN on a number of turbine design parameters are investigated and the position of the rotor relative to the tower (upwind or downwind rotor) is found to be the most important design parameter. For an upwind rotor the LFN levels are so low that it should not cause annoyance of neighbouring people. Important turbine design parameters with strong influence on LFN are the blade tip speed and the distance between rotor and tower.

\section{Introduction}

Today, thousands of wind turbines are installed per year worldwide. Typical sizes are from 1-3 MW nominal generator power and a rotor diameter ranging from 80-100 m but larger prototypes up to 5 MW and with a rotor diameter of $126 \mathrm{~m}$ have now been installed. One of the environmental issues is broadband noise from the rotors. This is typically noise from turbulent inflow and from the turbulent boundary layer at the trailing edge. However, recently there has been some concern on the level of low frequency noise (LFN) generated from these big installations.

In the early stage (around 1980-1990) of development of modern wind turbines, low frequency noise was experienced on the MOD-1 2 MW turbine ${ }^{1}$ and other downwind turbines in US ${ }^{2}$ as well as on the $3 \mathrm{MW}$ Maglarp turbine in Sweden ${ }^{3}$. On all turbines the noise generation was linked to the blade passing the unsteady wake behind the tower, Figure 1, which for the Swedish turbine was a tubular tower, whereas the MOD-1 turbine had a lattice tower with four main poles.

A summary of the low frequency noise results from 8 different turbines in US was presented by Sheperd and Hubbard ${ }^{4}$. They also present measurements of low frequency noise from two turbines with an upwind rotor and with a diameter of $43 \mathrm{~m}$ and $95 \mathrm{~m}$, respectively. The SPL level for the two turbines is considerably lower than for the downwind rotors and is described to be caused by irregularities in the inflow to the turbines such as terrain effects.

The objective of the present paper is to present an overview of LFN characteristics of modern MW turbines based on numerical simulations. The investigations will comprise the common upwind rotor concept but also the turbines with a downwind rotor will be included although this concept, as mentioned above, is known to generate LFN at a level that can cause annoyance of people living in the 
neighbourhood of the turbines. The reason to include the downwind rotor concept is that this turbine design has some advantages which could lead to future competitive designs compared with the upwind, three-bladed rotor. One of the main advantages of the downwind two-bladed rotor is that the use of a teetering hinge between the blades and the rotor shaft can effectively reduce the bending moments transferred to the shaft when compared with a rotor with a stiff hub. Finally, a free yawing or high flexible yawing rotor concept is easier to obtain on a downwind rotor due to the restoring yaw moment from the rotor thrust.

The organization of the paper is such that the model complex for simulations of LFN is first described. Then follows a section with results illustrating the quite different LFN characteristics of upwind and downwind rotors. More results illustrating the influence of different design parameters on LFN for downwind rotors are then presented and finally a few conclusions are made.

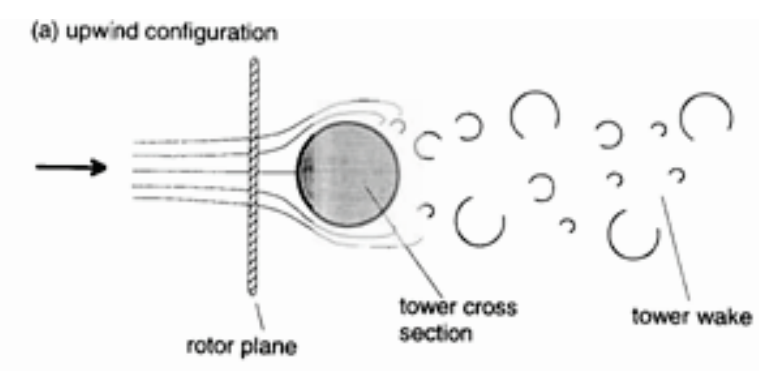

(b) downwind configuration

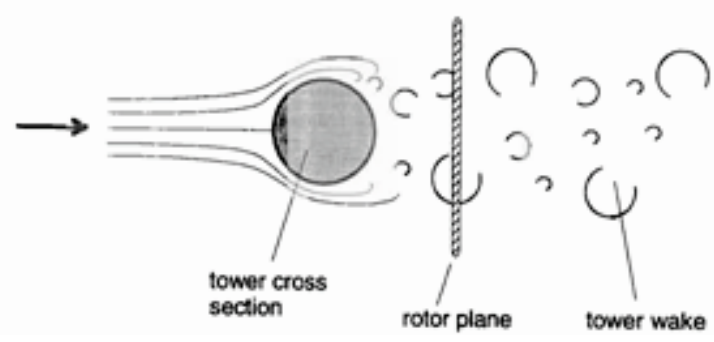

Figure $1 \quad$ The disturbed flow behind the tower results in highly unsteady aerodynamic blade forces which in the final end is the main cause of low frequency noise (illustration from Wagner ${ }^{5}$.

\section{The simulation model complex}

\subsection{The aeroacoustic model}

The research in US on low frequency noise from wind turbines performed in the period from the late seventies to the mid nineties led to the development of a model for computation of low frequency noise, the NASA-LeRC wind turbine sound prediction code by A. Viterna ${ }^{6}$. The model is based on established theories and methods for computation of propeller and compressor noise. In a compressor the rotating blades pass through the velocity deficits and disturbances of the stationary blades and this creates unsteady aerodynamic forces on the blades. Lowson ${ }^{7}$ has developed a general theory for such cases relating the sound pressure level (SPL) to the Fourier coefficients for the unsteady aerodynamic forces on the blades.

A number of assumption and simplifications have been made in the theory of Lowson in order to end up with a relatively compact model. One of the assumptions is to concentrate the unsteady aerodynamic forces on the blades at one radial station. However, for observation points not too close to the turbine this seems to be reasonable.

The implementation of the model follows the description by Viterna ${ }^{6}$ but is briefly described below. The RMS pressure variation of the $\mathrm{n}^{\text {th }}$ harmonic of the blade passage frequency is given by the following equations: 


$$
P_{n}=\frac{k_{n} \sqrt{2}}{4 \pi s}\left\{\sum_{p=1}^{\infty}\left[\begin{array}{l}
\left.e^{-i m(\Phi-\pi / 2)} J_{n B-p}\left(k_{n} r_{m} \sin \gamma\right) \cdot\left(a_{p}^{F} \cos \gamma-\frac{n B-p}{k_{n} r_{m}} a_{p}^{Q}\right)+\right] \\
e^{i m(\Phi-\pi / 2)} J_{n B+p}\left(k_{n} r_{m} \sin \gamma\right) \cdot\left(a_{-p}^{F} \cos \gamma-\frac{n B+p}{k_{n} r_{m}} a_{-p}^{Q}\right)
\end{array}\right]\right.
$$

where $k_{n}=\frac{n B \Omega}{c_{0}}$

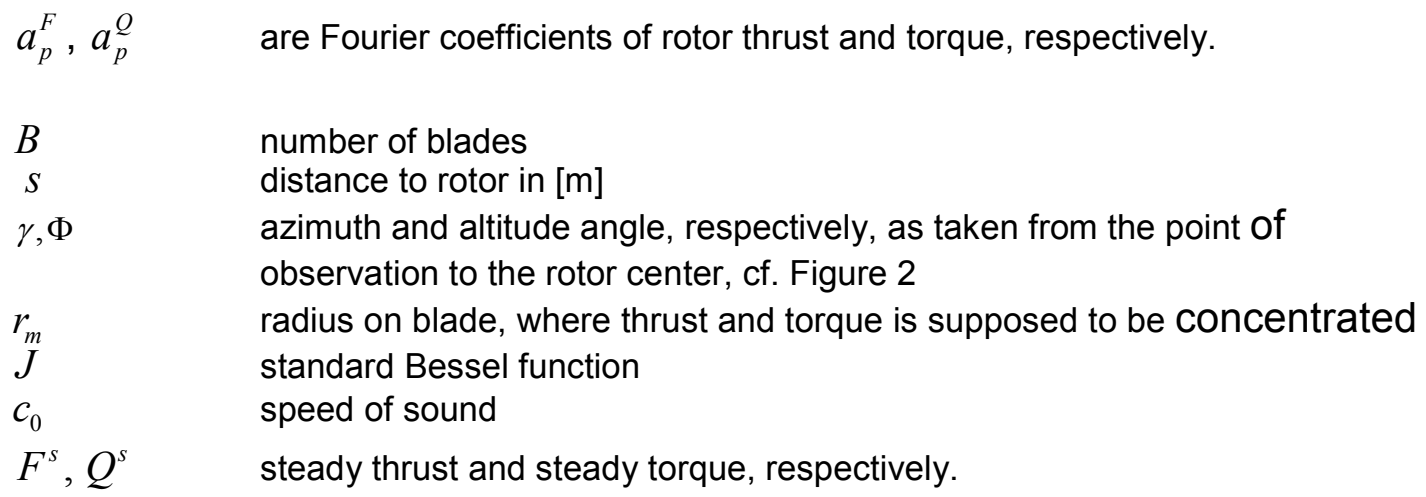

The Fourier coefficients of rotor thrust $a_{p}^{F}$ and rotor torque $a_{p}^{Q}$ are defined as:

$$
a_{p}^{F}=\frac{1}{T} \int_{0}^{T} F(\tau) e^{i p \frac{2 \pi}{T} \tau} d \tau
$$

and

$$
a_{p}^{Q}=\frac{1}{T} \int_{0}^{T} Q(\tau) e^{i p \frac{2 \pi}{T} \tau} d \tau
$$

where $F$ and $Q$ is rotor thrust and torque, respectively, and $T$ is the time for one rotor rev. However, in the present implementation we use a Fast Fourier Transform routine from the IMSL Math Library. Finally, the sound pressure level $S P L_{n}$ for each harmonic is computed as:

$$
S P L_{n}=10 \log _{10}\left(\frac{P_{n}^{2}}{P_{r e f}^{2}}\right) \text { where } \quad P_{r e f}=2 \cdot 10^{-5} \mathrm{~Pa}
$$

The total sound pressure level SPL is computed by summing up the $S P L_{n}$ from each harmonic:

$$
S P L=10 \log _{10}\left(\frac{1}{\hat{p}_{r e f}^{2}} \sum_{n} \hat{p}_{n}^{2}\right)
$$




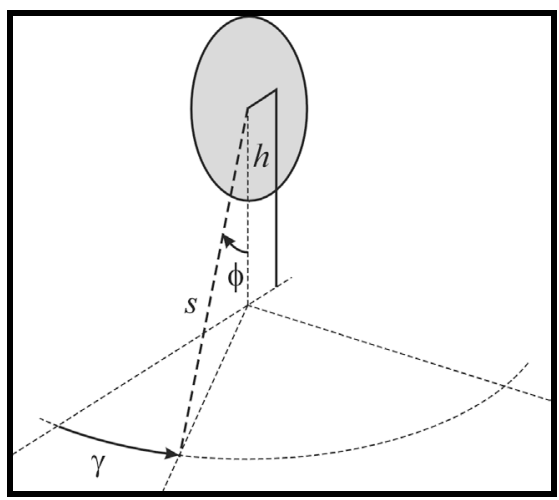

Figure 2. Definition of azimuth and altitude angles used in the acoustic model.

\subsection{Computation of the unsteady aerodynamic forces}

To compute the unsteady aerodynamic forces the general aeroelastic code HAWC2 developed at Risoe DTU is used. The HAWC2 code is a general model for time simulations of wind turbines and models the structural dynamics, the aerodynamics, the electrical system and the control system. The modelling of the tower disturbance on the flow is in particular important for the LFN computations. For an upwind rotor the tower disturbance flow model in HAWC2 is based on the potential flow model for a cylinder. For a downwind rotor the flow disturbance is computed with a model that is a combination of a source model that computes the initial velocity deficit from the tower based on the drag coefficient CD for the tower and for the development of this deficit further downstream the model equations are based on the boundary layer solution for a jet entering a fluid at still. More details on the tower flow models in HAWC2 have been presented by Madsen ${ }^{8}$. An example of computed velocity deficits are shown in Figure 3 for an upwind and a downwind rotor configuration. It is clearly
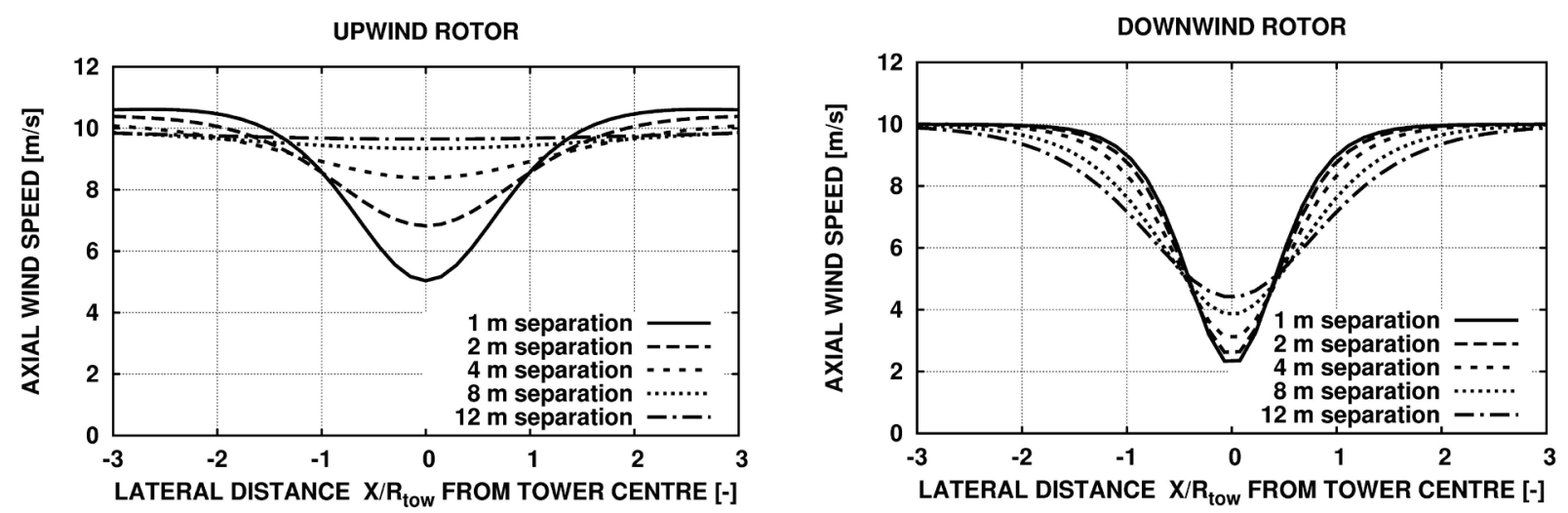

Figure 3 An example of computed influence on the flow velocity in the rotor plane from the tower, using the flow models in HAWC2 for an upwind rotor (left figure) and a downwind rotor (right figure). The tower diameter is $5.5 \mathrm{~m}$. and the undisturbed flow velocity is $10 \mathrm{~m} / \mathrm{s}$.

seen that the deficit from the downwind rotor is deeper and steeper than the deficit for the upstream rotor and it develops slower as function of increasing distance from the tower.

\subsection{The turbine models}

Two turbines, a three bladed turbine with a $127 \mathrm{~m}$ diameter upwind rotor and a two-blade turbine with a $127 \mathrm{~m}$ diameter downwind rotor, have been used in the present work. The turbines do not represent 
directly industrial designs but are close to the so-called $5 \mathrm{M}$ reference rotor $^{9}$, which has been used in different international research projects in the past.

Main turbine data:

- Blade length $63.5 \mathrm{~m}$

- Tower height $100 \mathrm{~m}$

- Tower diameter, $6 \mathrm{~m}$ at bottom, $4 \mathrm{~m}$ at top (diameter varied in simulations)

- No coning and no tilt

- Rotor speed $1.2 \mathrm{rad} / \mathrm{s}$ (parameter varied in simulations)

- Rated power $5 \mathrm{MW}$

- Stiff connection between shaft and rotor

The blades on the two-bladed rotor have a chord length that is $50 \%$ bigger relative to the three bladed rotor and the rotor then produce almost the same power as the three bladed. It should finally be noted that the simulations in the present case have been run for a structural stiff turbine model which means that the aeroelastic effects on turbine dynamics have been limited.

\subsection{The simulation procedure}

First we run a time simulation with HAWC2 and compute time traces of rotor thrust and rotor toque for a period of 70 sec., Figure 3. The Fourier Coefficients $a_{p}^{F}, a_{p}^{Q}$ are then computed using 8 segments of 1020 points and input to the aeroacoustic model which finally computes the RMS pressure variation of the $\mathrm{n}^{\text {th }}$ harmonic of the blade passing frequency. Finally, the sound pressure level SPL is computed using eq. 5. It has been chosen to present the SPL spectra without any weighting (A- weighting or $G$ weighting) as the focus in present paper is mainly on the LFN generation mechanisms and not on the annoyance and hearing thresholds.
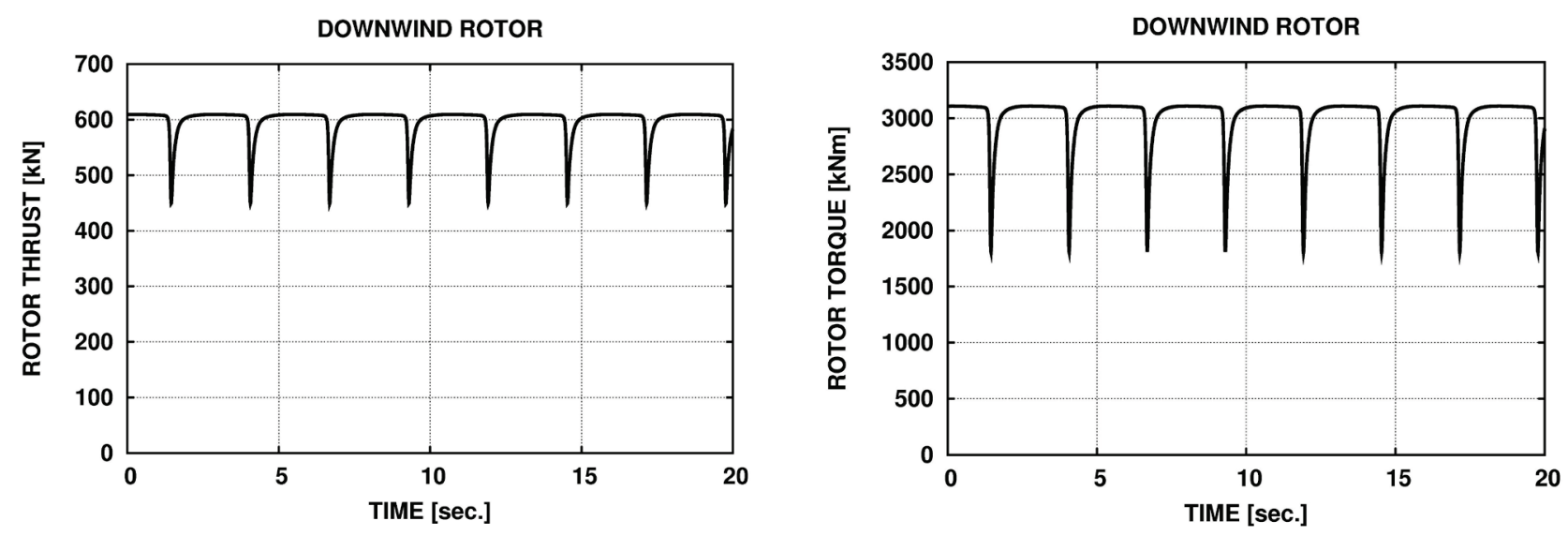

Figure $4 \quad$ An example of computed time traces with the aeroelastic code HAWC2 of rotor thrust and torque for the downwind rotor at a wind speed of $10 \mathrm{~m} / \mathrm{s}$.

\section{Broadband noise and low frequency noise}

The total noise from a wind turbine comprises broadband noise $(\mathrm{BN})$ as well as low frequency noise (LFN). Different noise sources contribute to the total BN noise but the two most important are turbulent trailing edge noise and noise from turbulent inflow. A common engineering model to compute $\mathrm{BN}$ for wind turbines is the model of Brooks, Pope and Marcolini ${ }^{10}$ (the BPM model). The result of a BN computation for the two-bladed turbine is shown in Figure 5 together with the results of computations of LFN. The listener position is somewhat arbitrarily chosen to $400 \mathrm{~m}$ downstream the rotor and this corresponds to four times the tower height. It is seen that just below $30 \mathrm{~Hz}$, LFN is dominant but this of 
course depends on the intensity of LFN and BN. It is standard today in industry to include broadband noise computations in the design process of wind turbines whereas this is not the case for LFN.

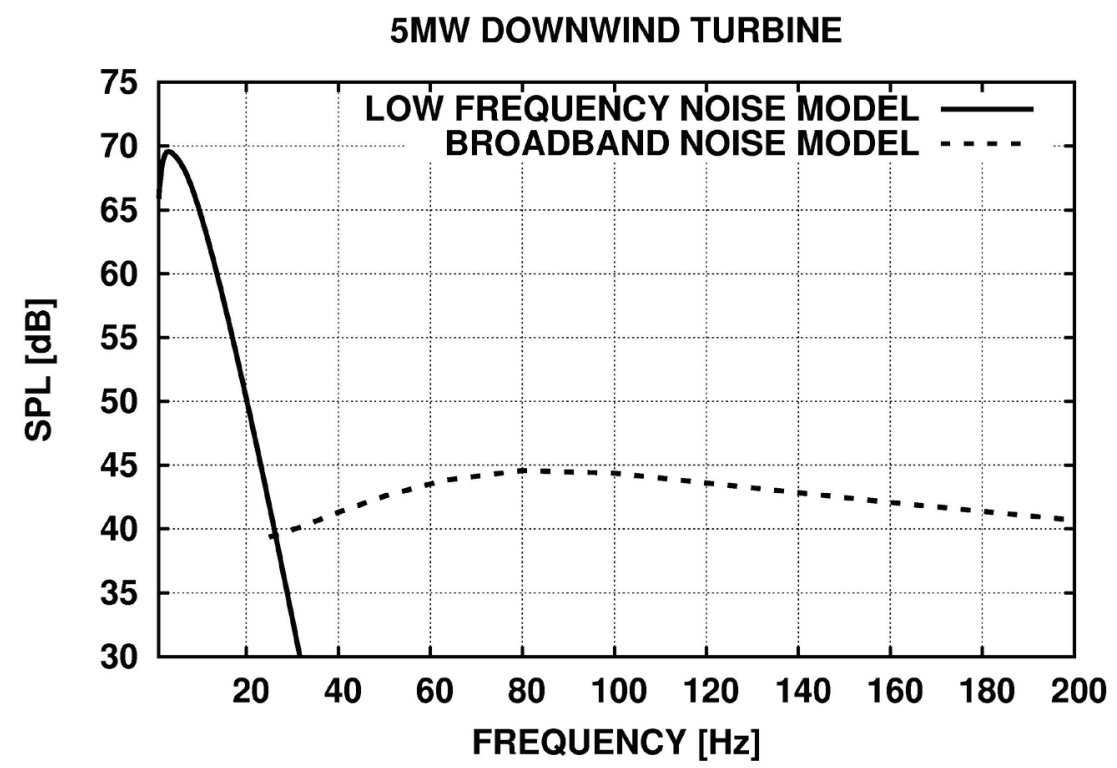

Figure $5 \quad$ An illustration of the total noise picture from a turbine originating from a broadband noise $(B N)$ prediction model and the present low frequency noise (LFN) model.

\section{Comparison of upwind and downwind rotor concepts}

The first major design parameter to investigate is the concept of having the rotor upwind or downwind the tower. As was illustrated in Figure 3, the velocity deficits from the tower are quite different and so are also the unsteady aerodynamic loads causing the LFN.

\subsection{Influence of distance between rotor and tower}

The difference between the concepts have been compared by computing LFN at a common operational wind speed of $10 \mathrm{~m} / \mathrm{s}$ and for different separations between rotor and tower. For a typical industrial rotor the minimum rotor/tower separation will probably not be less than 4-6 $\mathrm{m}$ but in the present case we have compared distances in the range from $1-12 \mathrm{~m}$, Figure 6 . It is clear from the figures that the distance
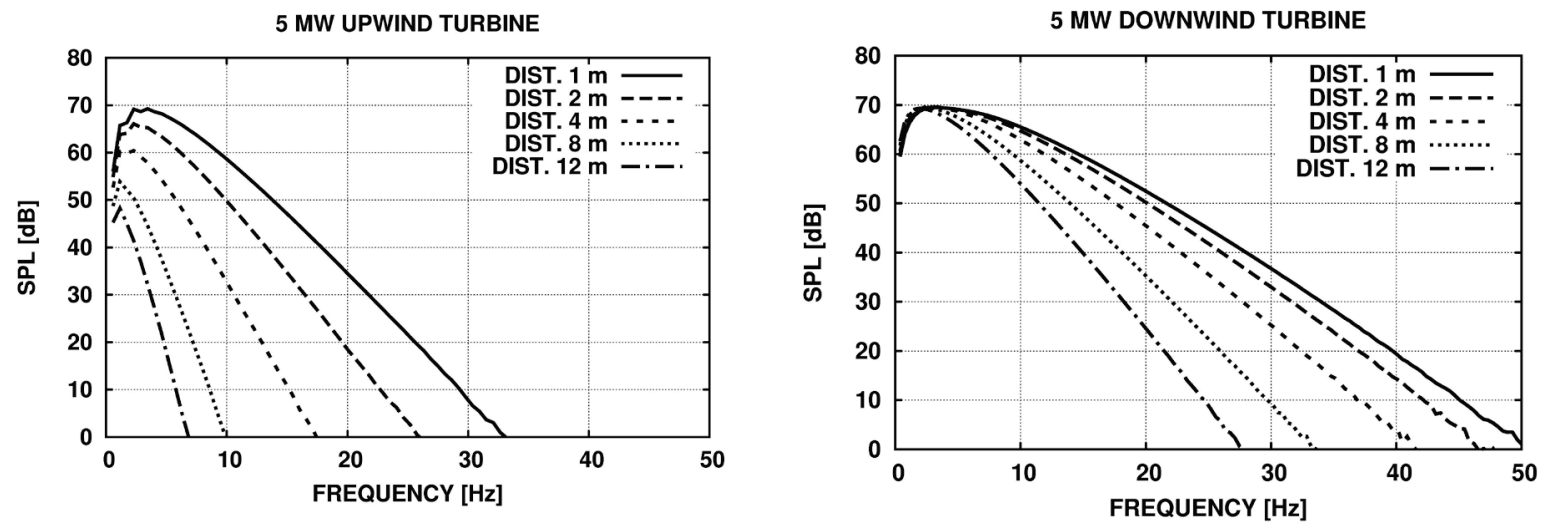

Figure 6 Comparison of SPL for an upwind and a downwind rotor, respectively, for different distances between tower wall and rotor plane. Wind speed is $10 \mathrm{~m} / \mathrm{s}$ and the listener position is $400 \mathrm{~m}$ downstream the turbine. 


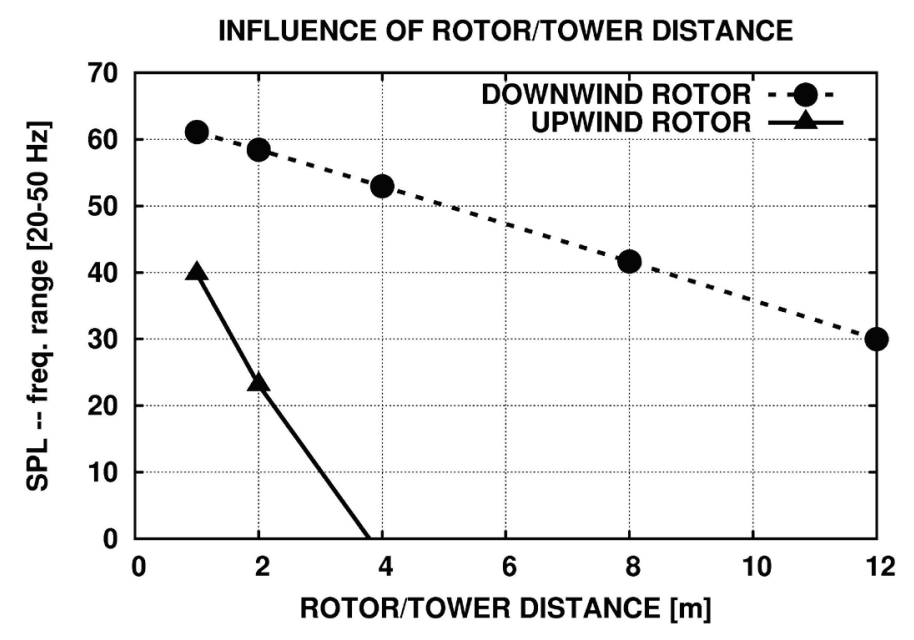

Figure $7 \quad$ Total SPL in the frequency range from 20-50 Hz for an upwind and a downwind rotor based on the spectra shown in Figure 6.

between the rotor and the tower is a very important parameter for the LFN level and this is summarized in Figure 7, where the total SPL level in the frequency range from $20-50 \mathrm{~Hz}$ is shown as function of rotor/tower distance for the two concepts. From this

figure it is clear that LFN in general should not be a problem for turbines with upwind rotors. In the following section we will therefore only show results of parameter variation for the downwind rotor concept.

\section{Influence of different operational parameters and design variables for the downwind rotor concept}

\subsection{Influence of distance to listener}

First we investigate the influence of distance to the listener. SPL was computed for four different distances, $200 \mathrm{~m}, 400 \mathrm{~m}, 800 \mathrm{~m}$ and $1600 \mathrm{~m}$ and it is seen that the spectra are almost identical but with decreasing level for increasing distance, left figure in Figure 8. The total SPL level in the frequency range from $20-50 \mathrm{~Hz}$ is shown as function of distance to listener in the right part of Figure 8 and it is seen, as expected, that SPL decreases with $6 \mathrm{~dB}$ for doubling the distance. However it should be noted that propagation effects from e.g. wind shear, turbulence and temperature gradients are not taken into account in the present modelling and this can change the damping of the noise considerably.

In the next sections, SPL will only be shown for a distance of $400 \mathrm{~m}$ from rotor centre to the listener.
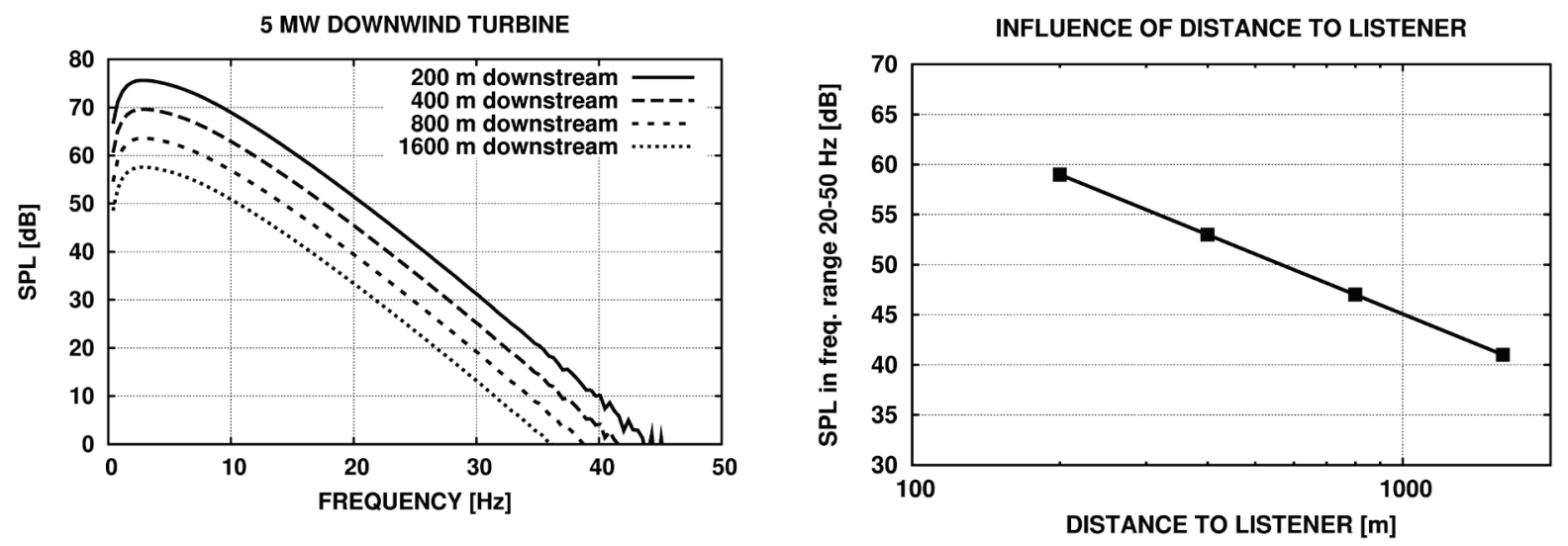

Figure $8 \quad$ Sound pressure level at different distances to listener. Simulation for downwind rotor, 10 $\mathrm{m} / \mathrm{s}$ and a rotor/tower distance of $4 \mathrm{~m}$. 


\subsection{Influence of wind speed on SPL}

Simulations have been run for wind speeds between 8 and $12 \mathrm{~m} / \mathrm{s}$ to investigate the influence of wind speed on LFN. From the results in Figure 9 it is clear that the LFN level is only a weak function of wind speed, about 3-4 dB for the wind speed variation from $8-12 \mathrm{~m} / \mathrm{s}$. However, most wind turbines today run with a variable rotor speed which is dependent on rotor power and thus in the final end of wind speed. The influence of the rotor speed will be investigated below.

\subsection{Influence of blade tip speed on SPL}

The rotor rotational speed for the simulations so far has been $1.2 \mathrm{rad} / \mathrm{s}$ giving a blade tip speed just below $70 \mathrm{~m} / \mathrm{s}$ and this is a typical value for industrial rotors. In the parameter variation the blade tip speed has now been varied from 63 to $82 \mathrm{~m} / \mathrm{s}$ and as can be seen in Figure 10 this has a considerable influence of the LFN. Again, the total SPL in the frequency range from $20-50 \mathrm{~Hz}$ has been derived and shown as function of blade tip speed. From this correlation it can be found that the SPL level is increased with around $0.8 \mathrm{~dB}$ for an increase of tip speed with $1 \mathrm{~m} / \mathrm{s}$. Rotor speed is thus an important design parameter for control of LFN. This is also the case for broadband noise where noise limitations have led to a typical tip speed around 70

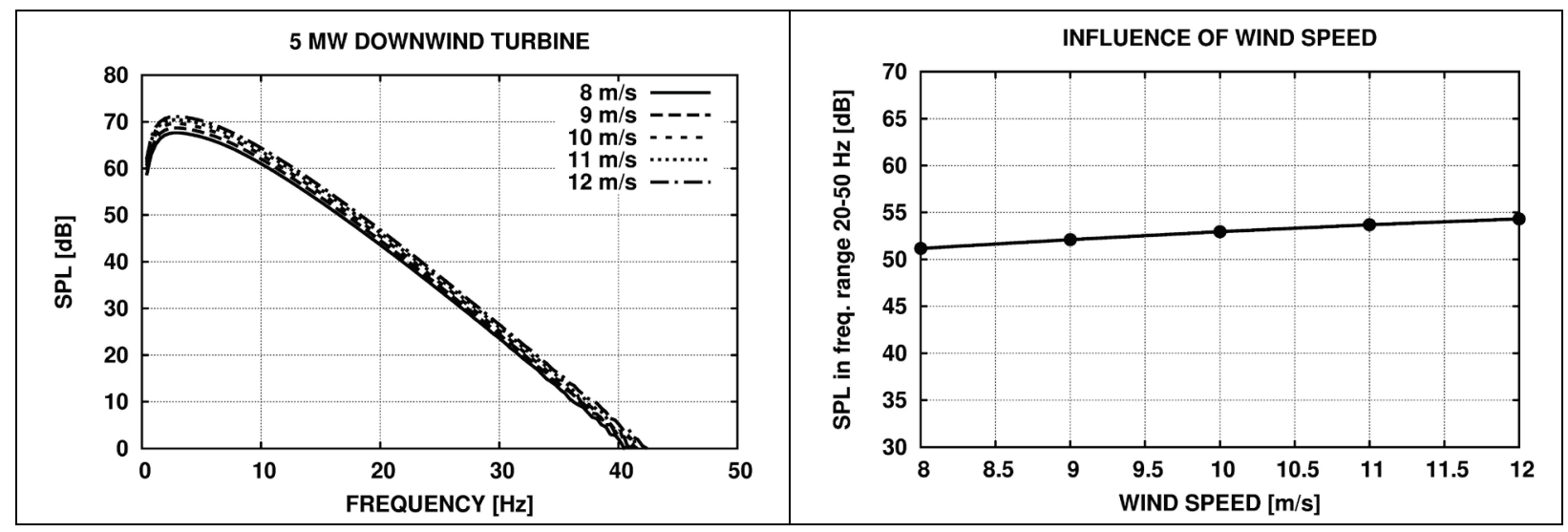

Figure $9 \quad$ The SPL as function of wind speed for a listener position $400 \mathrm{~m}$ downstream.
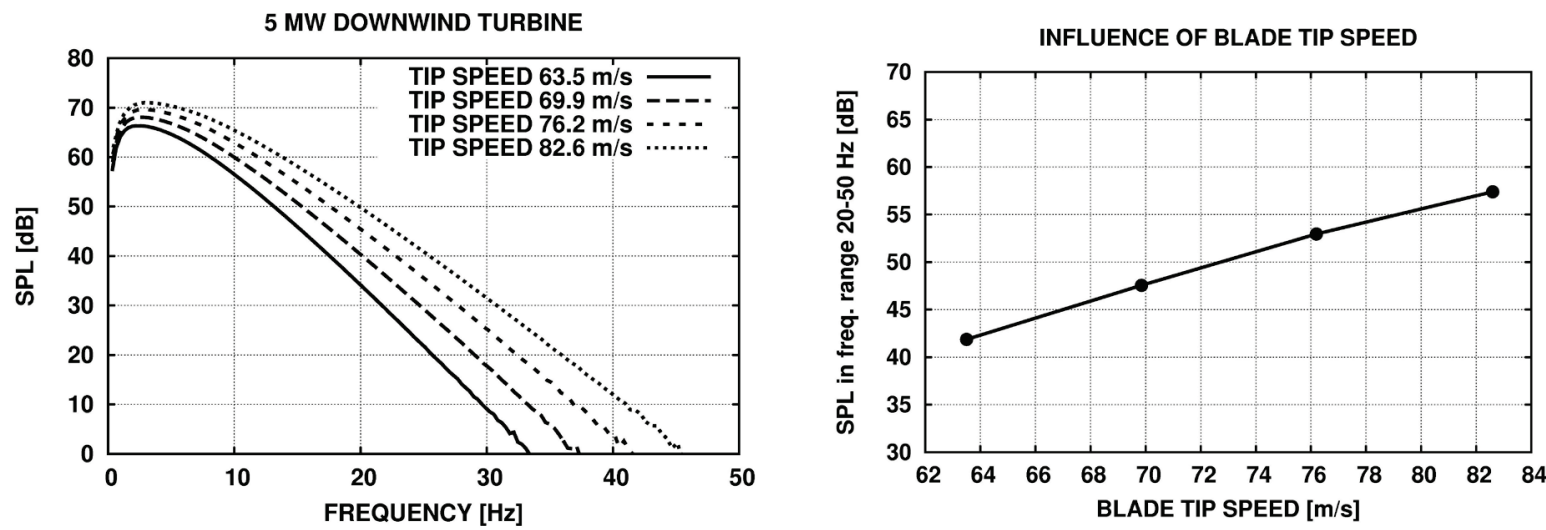

Figure $10 \quad$ The SPL as function of blade tip speed at a wind speed of $10 \mathrm{~m} / \mathrm{s}$ and for a listener position $400 \mathrm{~m}$ downstream.

$\mathrm{m} / \mathrm{s}$. If there were no noise restriction we would probably see somewhat higher tip speeds on the turbines as this can lead to more slender blades and thus material savings.

\subsection{Influence of tower drag coefficient on SPL}

The drag coefficient $C D$ for the tower is not really a free design parameter as most turbine towers are cylindrical and the minimum drag coefficient is a function of 

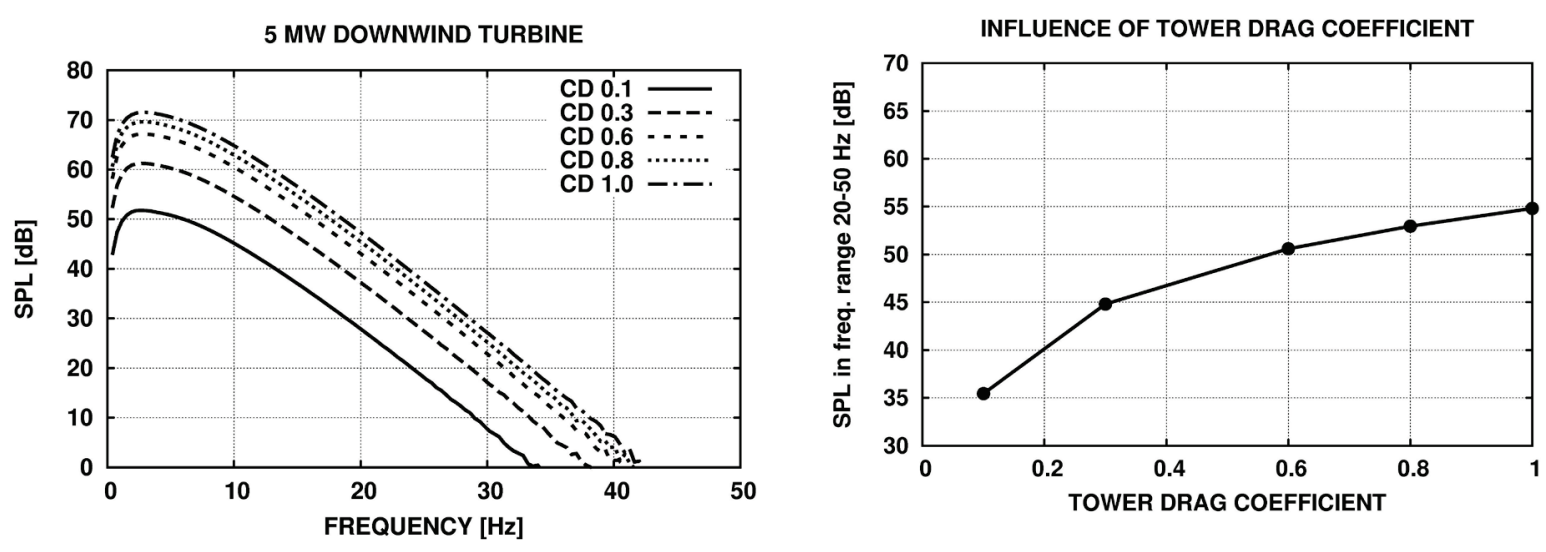

Figure 11

The SPL as function of the tower drag coefficient at a wind speed of $10 \mathrm{~m} / \mathrm{s}$ and for a listener position $400 \mathrm{~m}$ downstream.

Reynolds number and of course of the smoothness of the tower surface. In the simulations so far a drag coefficient of 0.8 has been used and this is probably in the high range for a cylinder with a Reynolds number of 5 mill. which is the value for the present tower.

In the parameter variation performed we have varied the drag coefficient between 0.1 and 1.0 and from the LFN results in Figure 11 it is seen that within a realistic range of CD from e.g. 0.6 to 0.9 the increase in LFN with CD is moderate, about an increase of $0.9 \mathrm{~dB}$ for an increase in $\mathrm{CD}$ of 0.1 .

\subsection{Influence of tower diameter on SPL}

The final design parameter we have investigated is the tower diameter although this again is not a free parameter due to constraints from stress limitations in the tower walls. So far the tower diameters have been $6 \mathrm{~m}$ at the bottom and $4 \mathrm{~m}$ at the top. A variation is now performed from 4-7 $\mathrm{m}$ at the bottom and 2-5 $\mathrm{m}$ at the top. The influence on the LFN is somewhat surprising as the SPL level in most of the considered frequency range decreases as function of increasing tower diameter as can be seen in Figure 12. To investigate this, the velocity deficit from the smallest and biggest diameters were plotted, Figure 13. Increasing the tower diameter the deficit becomes deeper as the distance between rotor and tower centre is kept constant. This should increase the LFN level but it is also clear that the gradients of the lift on the blade as function of time shown in the right figure in Figure 13 decreases and this effect most be stronger so that overall the LFN decrease with increasing tower diameter.
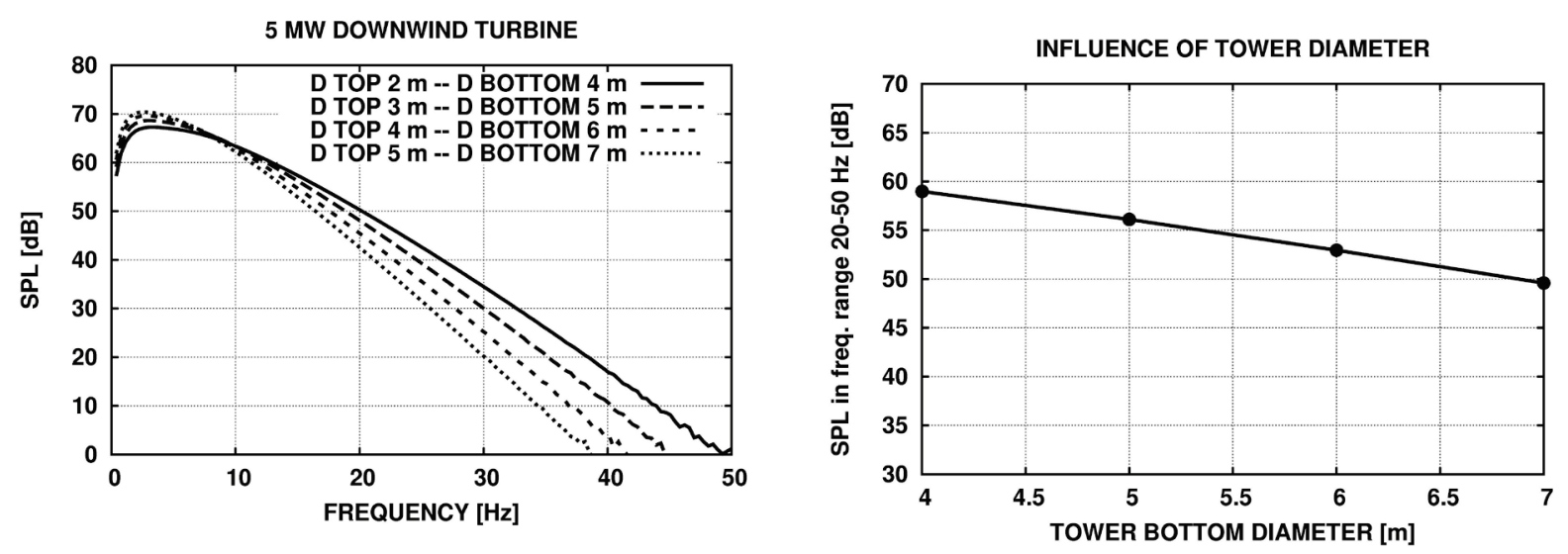

Figure 12

The SPL as function of the tower drag coefficient at a wind speed of $10 \mathrm{~m} / \mathrm{s}$ and for a listener position $400 \mathrm{~m}$ downstream. 

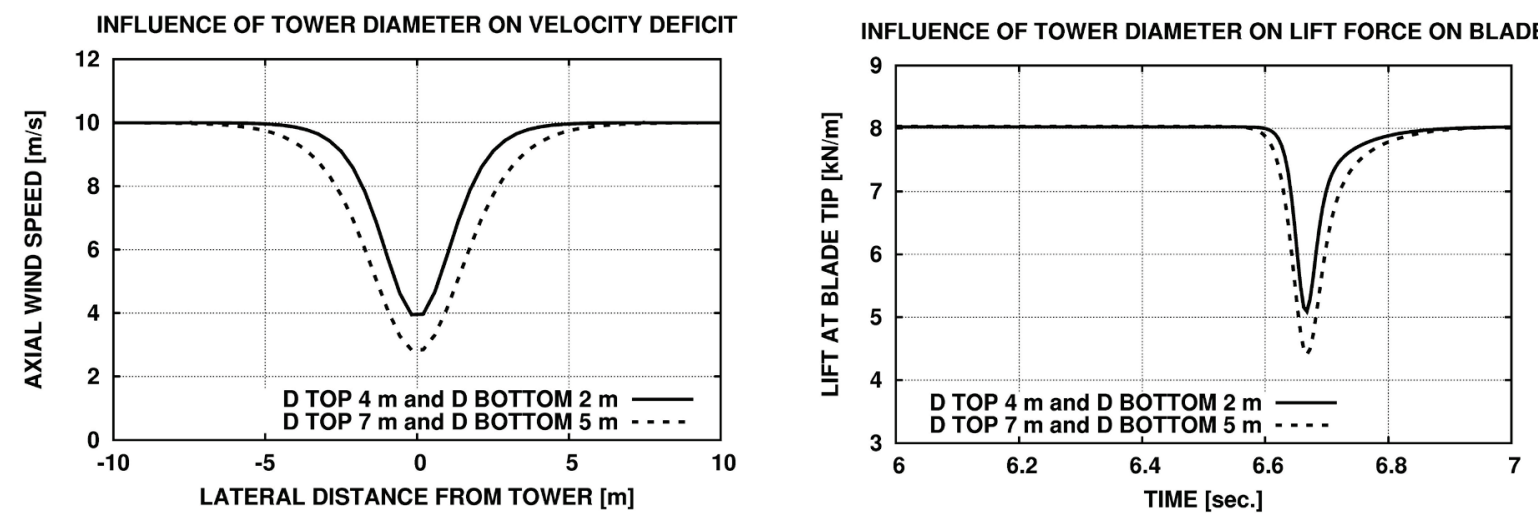

Figure 13 The SPL as function of the tower drag coefficient at a wind speed of $10 \mathrm{~m} / \mathrm{s}$ and for a listener position $400 \mathrm{~m}$ downstream.

\subsection{Influence of other parameters}

In the present investigation the wake flow deficit has been computed with a steady model but if no specific design considerations are taken to prevent vortex shedding

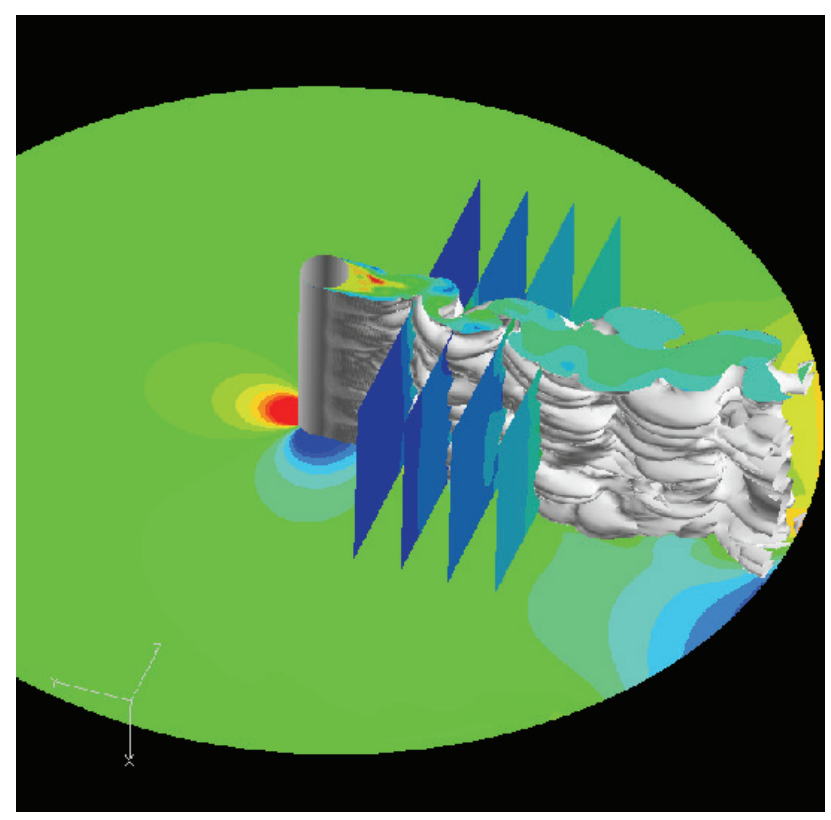

Figure 14 CFD computation of the unsteady flow in the wake of a cylinder. Iso-vorticity surfaces are shown and in the plane perpendicular to the cylinder are shown pressure contours. From Madsen ${ }^{8}$.

from the cylindrical tower, e.g. mounting a spiral surface, vortex shedding will probably occur and the wake flow becomes highly unsteady. In a recent investigation ${ }^{8}$ it was shown that the vortex shedding as shown in Figure 14, can increase the LFN SPL level with as much as 5-20 dB.

Another source of increase of LFN is shear in the inflow to the rotor. Considerable shear is seen in inflow on MW rotors as their blades from top position to bottom position span a considerable height. In the present case the blade tip in top position is at a height of $163 \mathrm{~m}$ and in the bottom position is at a height of just $37 \mathrm{~m}$. The inflow wind speed in these two heights can be quite different and this leads to unsteady forces on the blades and thus a source for LFN. Even stronger shear profiles and more unsteady aerodynamic forces occur when a turbine operates in half wake of an upstream turbine and this will also be a source of increased LFN.

Finally, turbulence in the inflow to the rotor will contribute to the LFN level but the noise from inflow turbulence is normally also modelled with the broadband noise prediction models for wind turbines as the BPM model implemented by Fuglsang end Madsen ${ }^{11}$. 


\section{Annoyance of people from LFN}

We will not in this paper go into details with the subject of annoyance of people from LFN but just present two figures on LFN hearing thresholds. The first one, Figure 15, is from the work of Birgitta Berglund et. al. ${ }^{12}$ showing measured hearing thresholds for LFN. Figure 16 is from a more recent report by Leventhall ${ }^{13}$ who discusses the hearing thresholds in details. Besides measured thresholds, the ISO 389-7 threshold standard is also shown in Figure 16. In general comparing the computed LFN levels in the present paper with these threshold values, most of the investigated cases show LFN levels considerably below the threshold values. On the other hand it has been mentioned that the influence from unsteadiness of the flow behind the tower such as vortex shedding may increase the levels with $5-20 \mathrm{~dB}$ and then the computed levels certainly could be above the threshold levels.

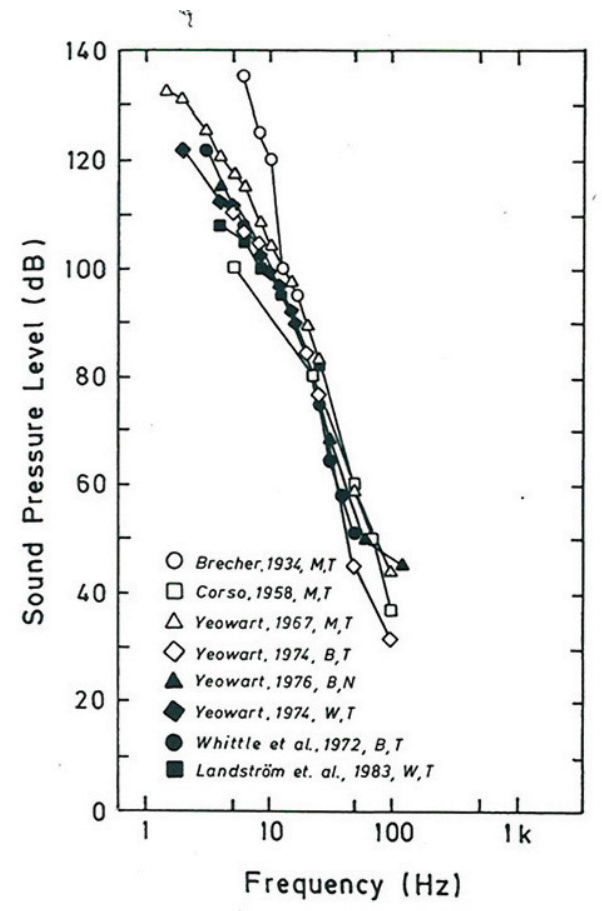

FIG. 2. Hearing thresholds as a function of signal frequency in various studies $(\mathrm{M}=$ monaural; $\mathrm{B}=$ binaural; $\mathrm{W}=$ whole body; $\mathrm{T}=$ tone; $\mathrm{N}=$ noise

band).

Figure $15 \quad$ Measured hearing thresholds for LFN as function of frequency. From paper of Birgitta Berglund et. al. ${ }^{12}$.

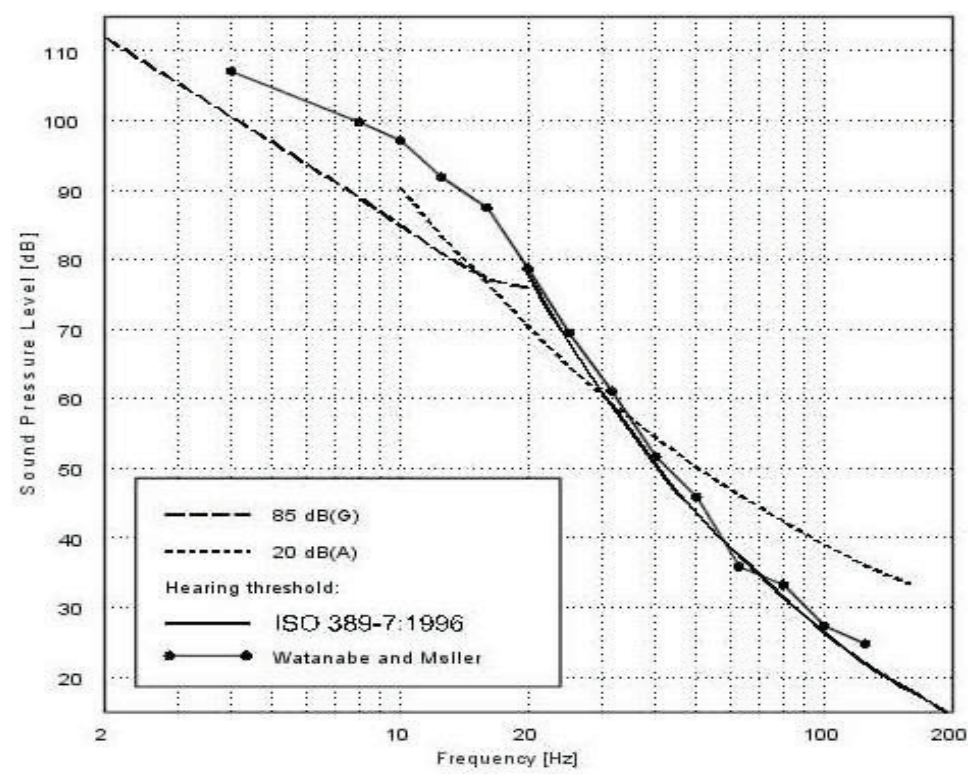

Figure 16 Different LFN hearing tresholds. Figure from report of Leventhall ${ }^{13}$. 


\section{Conclusions}

The objective of the present paper has been to present an overview of LFN characteristics of modern MW turbines based on numerical simulations. The following main findings have been achieved:

- The design concept of having the rotor upstream or downstream the tower is the most important parameter for the level of LFN. A downstream rotor has LFN levels that are $20 \mathrm{~dB}$ or higher compared with upstream rotors

- Blade tip speed is a very important design parameter and we found an increase in LFN level of $0.8 \mathrm{~dB}$ for an increase of tip speed of $1 \mathrm{~m} / \mathrm{s}$

- The distance between the rotor plane and the tower is important with a correlation of $2.8 \mathrm{~dB}$ reduction of LFN for an increase of distance with $1 \mathrm{~m}$

- LFN increases only slightly with tower drag coefficient

- It was found that LFN decreases slightly with increasing the tower diameter and this seems to be due to two counteracting effects where the gradients of the velocity deficit is more important than the depth of the deficit.

\section{Acknowledgement}

The research work has been carried out under two contracts under the Danish Energy Research program EFP:

Contract: J.nr.: 033001/33032-0081

EFP-2006 project "Lavfrekvent støj fra store vindmøller-kvantificering af støjen og vurdering af genevirkningen” "

Contract: J.nr.: 033011/33033-0266

Program for Forskning i Anvendt Aeroelasticitet.

\section{References}

1 Kelly, N.D. "Acoustic Noise generation by the DOE/NASA MOD-1 Wind Turbine". Proceedings of Workshop "Wind Turbine Dynamics" held at Cleveland State University, Cleveland, Ohio, February 2426, 1981. pp. 375-387.

2 Hubbard, H. H. and Shepherd, K. P., "Aeroacoustics of large wind turbines," Journal of Acoustical Society of America 89 (6) June 1991, pp. 2495-2508.

3 Ljungren, S. "A Preliminary Assessment of Environmental Noise from large WECS, based on Experiences from Swedish Prototypes". Report FFA TN 1984-48.

4 Shepherd, K.P. and Hubbard, H.H. "Physical Characteristics and Perception of Low Frequency Noise from Wind Turbines". Noise Control Engineering Journal, Volume 36 Number 1, January February 1991.

5 Wagner, S., Bareiss, R., Guidatti, G. "Wind Turbine Noise”. Springer -Verlag Berlin Heidelberg 1996. European Commission (DGXII), EUR 16823.

6 Viterna, L.A. "Method for Predicting Impulsive Noise Generated by Wind Turbine Rotors". Presented at Second DOE/NASA Wind Turbine Dyn. Workshop, Cleveland, 1981.

7 Lowson, M.V. "Theoretical Analysis of Compressor Noise Evaluation". J. Acoust. Soc. Am. , 47, 1 (part 2), pp 371-385, 1970. 
8 Madsen, H.A., Johansen, J., Sørensen, N.N., Larsen, G.C. and Hansen, M.H. "Simulation of Low frequency Noise from a Dowwind Wind Turbine Rotor". Paper AIAA 2007-623 presented at 45th AIAA Aerospace Sciences Meeting and Exhibit, 8-11 January 2007, Reno, Nevada, US.

9 Jonkman, J. "IEA Annex XXIII document on NREL's baseline wind turbine aeroelastic model", NREL/NWTC, August 2005.

10 Brooks, T.F., Pope, D.S. and Marcolini, M.A., 1989, "Airfoil Self-Noise and Prediction", NASA Reference Publication 1218, National Aeronautics and Space Administration, USA.

11 Fuglsang, P.; Aagaard Madsen, H., Implementation and verification of an aeroacoustic noise prediction model for wind turbines. Risø-R-867(EN) (1996) 53 p.

12 Berglund,B., Hassmén, P. and Soames Job, R.F. "Sources and effects of low frequency noise". J. Acoust. Soc. Am. 99 (5), May 1996. pp. 2985-3002.

13 Leventhall, G. "A Review of Published Research on Low Frequency Noise and its Effects". May 2003, DEFRA 1/2/50. 